\title{
Quantitative Evaluation of Artifact Removal in Real Magnetoencephalogram Signals with Blind Source Separation
}

\author{
Javier Escudero, ${ }^{1,2}$ Roberto Hornero, ${ }^{2}$ Daniel Abásolo,,${ }^{2,3}$ and Alberto Fernández ${ }^{4,5}$ \\ ${ }^{1}$ Signal Processing and Multimedia Communications; School of Computing and Mathematics, University of Plymouth, \\ Plymouth PL4 8AA, UK; ${ }^{2}$ Biomedical Engineering Group, E.T.S.I. Telecomunicación, University of Valladolid, Valladolid, \\ Spain; ${ }^{3}$ Centre for Biomedical Engineering; Division of Mechanical, Medical \& Aerospace Engineering; Faculty of Engineering \\ and Physical Sciences, University of Surrey, Guildford GU2 7XH, UK; ${ }^{4}$ Departamento de Psiquiatría y Psicología Médica, \\ Universidad Complutense de Madrid, Madrid, Spain; and ${ }^{5}$ Laboratorio de Neurociencia Cognitiva y Computacional, Centro de \\ Tecnología Biomédica, Universidad Politécnica de Madrid y Universidad Complutense de Madrid, Madrid, Spain
}

\begin{abstract}
The magnetoencephalogram (MEG) is contaminated with undesired signals, which are called artifacts. Some of the most important ones are the cardiac and the ocular artifacts (CA and OA, respectively), and the power line noise (PLN). Blind source separation (BSS) has been used to reduce the influence of the artifacts in the data. There is a plethora of BSS-based artifact removal approaches, but few comparative analyses. In this study, MEG background activity from 26 subjects was processed with five widespread BSS (AMUSE, SOBI, JADE, extended Infomax, and FastICA) and one constrained BSS (cBSS) techniques. Then, the ability of several combinations of BSS algorithm, epoch length, and artifact detection metric to automatically reduce the CA, OA, and PLN were quantified with objective criteria. The results pinpointed to cBSS as a very suitable approach to remove the CA. Additionally, a combination of AMUSE or SOBI and artifact detection metrics based on entropy or power criteria decreased the OA. Finally, the PLN was reduced by means of a spectral metric. These findings confirm the utility of BSS to help in the artifact removal for MEG background activity.
\end{abstract}

Keywords-Blind source separation, Cardiac artifact, Evaluation, Independent component analysis, Magnetoencephalogram, Ocular artifact, Power line noise.

\section{INTRODUCTION}

The magnetoencephalogram (MEG) is the noninvasive recording of the magnetic fields generated by the neurons. Likewise the electroencephalogram
(EEG), it is a neurophysiological technique able to directly measure brain activity. Although both EEG and MEG provide high temporal resolution, the latter offers some advantages over the former. The magnetic recordings do not depend on any reference point and they are less affected by extra-cerebral tissues than the EEG. ${ }^{18}$ However, bodily organs contaminate the recordings with undesired activity (known as artifacts) that can bias the analyses. ${ }^{18}$

Normal brain activity usually generates magnetic fields whose amplitude ranges in the order of hundreds of $\mathrm{fT}$. On the other hand, the heart produces much stronger magnetic fields, which have a considerable effect on the recordings of spontaneous brain activity. This is known as cardiac artifact (CA). ${ }^{23}$ Significant contamination is also caused by eye blinks and movements. ${ }^{1}$ Each eye can be modeled as a small electrical dipole. ${ }^{6}$ Eye movements and blinks modify the orientation and intensity of such a dipole, thus altering the associated electromagnetic fields near the eyes and causing the ocular artifact (OA). ${ }^{6}$ Apart from these biological activities, the power line noise (PLN) is also a noticeable source of contamination in brain recordings. ${ }^{12,25}$ The removal of the PLN with notch filters is not a satisfactory solution if its frequency overlaps with that of the signals. ${ }^{18}$

Several methods have been tested to remove the artifacts from EEG and MEG data. These include: epoch rejection, ${ }^{14}$ regression techniques, ${ }^{6}$ and blind source separation (BSS). ${ }^{22,39}$ The simplest method to eliminate the artifacts is epoch rejection, which discards raw data epochs with high artifactual contamination. This is performed individually for each epoch, which is time-consuming. ${ }^{14}$ If the contamination is 
widespread, epoch rejection produces significant data loss. This method is intrinsically subjective. ${ }^{14,25}$ Another method to attenuate some artifacts in the MEG and, especially, in the EEG is removing the projection of an auxiliary signal from the brain recordings. ${ }^{14,25}$ This technique has been widely applied to remove the OA from the EEG. ${ }^{6}$ It consists of computing the projection of the reference signal on the recordings and then subtracting it from the data. ${ }^{6}$ Nevertheless, this technique introduces new unexpected artifacts in the recordings if the reference signal (most often the electrooculagram, EOG) contains some brain activity. ${ }^{14,25}$ Furthermore, it requires to record the auxiliary signal and the brain data simultaneously. ${ }^{14,25}$

BSS $^{22,39}$ has also been used in the artifact removal problem. ${ }^{12,25,34,36}$ BSS estimates the set of components, or sources, which can be linearly combined to obtain the measured signals. BSS does not need previous information about the recordings and its assumptions are suitable for MEG and EEG data. ${ }^{22,39}$ Nonetheless, the use of a reference, if available, helps to guide the extraction of the artifacts with a constrained blind source separation (cBSS). ${ }^{21}$

A major problem in this BSS-based artifact removal is the detection of the artifactual components. BSS isolates the artifacts into just a few sources, ${ }^{22}$ but the visual inspection of the contaminating activity is timeconsuming. Thus, several studies have introduced criteria to detect the artifacts. For instance, the use of statistical metrics (e.g., kurtosis and entropy) has been proposed to identify undesired components in EEG and MEG. ${ }^{2,7,9,12,27,31}$ In addition to being used in cBSS,${ }^{21}$ reference signals help to identify the artifactual sources by means of measures like correlation. ${ }^{2,7,24}$ The scalp topography can also be used to remove OAs since most ocular activity is located near the eyes. ${ }^{12,28,32,34}$ In some cases, such as OAs and PLN, spectral features of the components are helpful in the identification of the artifacts. ${ }^{24,27,33}$ Some of those criteria have been combined to detect various types of artifacts. ${ }^{2,12,24,27,34,35}$

Despite the number of metrics proposed to detect artifactual activity in brain signals, $2,7,9,12,24,27,28,31,32,3436$ few comparative studies are available. Moreover, the evaluation of the artifact rejection has quite often been made on subjective bases or with synthetic signals. Very few studies have quantified the level of artifacts in real recordings before and after the artifact removal. ${ }^{7,12}$ Finally, most of those efforts have been devoted to the OA in the EEG, ${ }^{24,34} 36$ with the MEG receiving less attention. 7,12,31

Thus, we have applied diverse straightforward BSS approaches to remove the CA, OA, and PLN. Then, we have objectively compared their performance for real MEG recordings. Those simple detection criteria had previously been proposed elsewhere (see, for instance, Refs. 2, 7, 12, 17, 30,31,35) and they are here tested on a different dataset. In contrast to other studies, this study applies and tests the BSS-based artifact removal on real brain signals without the need for simultaneous acquisitions of auxiliary signals.

\section{SUBJECTS AND MEG RECORDING}

Twenty-six healthy elderly subjects took part in the study ( 9 men and 17 women). The average age was $71.77 \pm 6.38$ years (mean \pm standard deviation, SD). The subjects are part of a larger database collected to analyze the impact of Alzheimer's disease on the $\mathrm{MEG}^{11,13}$ and they have been described elsewhere. ${ }^{10}$ All participants gave their informed consent for the research study, which was approved by the local ethics committee.

The MEG signals were recorded in a magnetically shielded room with a 148-channel whole-head magnetometer (MAGNES $2500 \mathrm{WH}, 4 \mathrm{D}$ Neuroimaging) in the MEG Center Dr. Pérez-Modrego (Complutense University of Madrid, Spain). The recordings were acquired while the subjects were lying on a patient bed with eyes closed in a relaxed state. To mimic the conditions of clinical studies, they were asked to stay awake and not to move eyes and head. For each subject, $5 \mathrm{~min}$ of $\mathrm{MEG}$ recording were acquired at a sampling rate of $169.54 \mathrm{~Hz}$. Afterwards, a bandpass FIR filter with cut-off frequencies at 0.5 and $60 \mathrm{~Hz}$ was applied to the MEGs, which were finally split into epochs of $10,20,40,60$, and $90 \mathrm{~s}(1695,3390,6780$, 10170 , and 15255 samples, respectively).

\section{METHODS}

\section{BSS for Artifact Removal}

BSS denotes a set of data-driven approaches to extract the underlying sources, or components, from a number of measurements. The term Independent component analysis (ICA) is often used to refer to some of the BSS techniques. ${ }^{22,39}$ BSS represents a set of $m$ measured time-varying signals, $\mathbf{x}(t)=\left[x_{1}(t), \ldots\right.$, $\left.x_{m}(t)\right]^{\mathrm{T}}$, where ${ }^{\mathrm{T}}$ denotes transposition, as an unknown linear mixture of $l$ latent underlying components, $\mathbf{s}(t)=\left[s_{1}(t), \ldots, s_{l}(t)\right]^{\mathrm{T}}$, given by a full-rank $m \times l$ mixing matrix, A..$^{2,39}$ A vector $\mathbf{n}(t)=\left[n_{1}(t), \ldots\right.$, $\left.n_{m}(t)\right]^{T}$ can also be included in the model to account for additive noise. ${ }^{10,12,22,36}$ The purpose of this additive noise term is twofold. It accounts for sensor noise corrupting the measurements ${ }^{22}$ and for the modeling error of the BSS estimation. ${ }^{36}$ Thus, the BSS model becomes: 


$$
\mathbf{x}(t)=\mathbf{A s}(t)+\mathbf{n}(t) .
$$

Since only the observations, $\mathbf{x}(t)$, are available, several assumptions are needed to estimate $\mathbf{A}$ and $\mathbf{s}(t)$ and to deal with $\mathbf{n}(t)$. In addition to linearity, it is hypothesized that $m \geq l$ and that the mixture is stationary. Moreover, the components are assumed to be mutually independent or, alternatively, decorrelated at any time delay. ${ }^{22,39}$ All these hypotheses have been validated for brain signals. ${ }^{22,39}$ Using these assumptions, the estimated components, $\hat{\mathbf{s}}(t)$, are computed as:

$$
\hat{\mathbf{s}}(t)=\mathbf{B} \mathbf{x}(t)=\mathbf{B}[\mathbf{A} \mathbf{s}(t)+\mathbf{n}(t)]=\mathbf{s}(t)+\mathbf{B n}(t),
$$

where $\mathbf{B}$ is the BSS demixing matrix, which must fulfill $\mathbf{B}=\hat{\mathbf{A}}^{+}$. Here, ${ }^{+}{ }^{\text {and }}{ }^{\wedge}$ denote pseudo-inverse matrix and estimated variable, respectively. ${ }^{22,39}$

The components are inspected to find out which ones are responsible for the artifacts. ${ }^{2,12,36}$ Once the artifactual components have been identified, the clean signals are reconstructed by subtracting the artifactual components from the recordings:

$$
\mathbf{x}_{\text {clean }}(t)=\mathbf{x}(t)-\sum_{j \in J} \hat{\mathbf{a}}_{j} \hat{\mathbf{s}}_{j}(t),
$$

where $J$ denotes the indices of all artifactual components and $\hat{\mathbf{a}}_{j}$ is the corresponding column of $\hat{\mathbf{A}}$. This approach is adopted to minimize the distortion of the true brain activity. ${ }^{36}$ Moreover, Eq. (3) can also be used with a cBSS algorithm, which extracts only the BSS component of interest. ${ }^{19}$

\section{Preprocessing and Model Order Selection}

The implementation of most BSS algorithms assumes a noiseless mixture where $m=l .^{22}$ However, EEG and MEG are affected by additive noise that corrupts the measurements of the weak electromagnetic fields. Furthermore, the number of channels in current EEG and MEG systems can be much larger than that of meaningful BSS components (i.e., $m>l$ ). Hence, a suitable preprocessing is needed to reduce the importance of the measurement noise and the dimensionality of the input signals in the BSS algorithm. ${ }^{10,12,22} \mathrm{We}$ apply a preprocessing based on factor analysis (FA). This approach is robust to the presence of additive noise of different power at each sensor. The model order $(l)$ is estimated with a statistical criterion: the minimum description length (MDL). ${ }^{12}$ The preprocessing matrix for whitening and dimension reduction and the additive noise power are estimated iteratively for several possible values of $l$. This consists of iteratively computing the preprocessing matrix from the eigenvalue decomposition of the recordings' covariance matrix minus the current estimation of the additive noise power and then re-estimating the noise power from the lack of fit of the diagonal elements of the covariance matrix. ${ }^{12}$ Once the preprocessing matrix and the noise power estimations have converged, the MDL value is computed for the corresponding $l .^{12}$ The optimum number of components is selected as the one providing the minimum MDL and the preprocessing matrix calculated for that value of $l$ is used to process the recordings. Further details about this preprocessing can be found elsewhere. ${ }^{10,12}$

\section{BSS Algorithms}

In addition to one cBSS technique, ${ }^{19,21}$ we compared five BSS algorithms commonly used in the analysis of EEGs and MEGs - AMUSE, SOBI, JADE, extended Infomax (eInfomax), and FastICA as shown by several publications on the use of BSS for EEG and MEG. ${ }^{9} 11,13,15,22,40$ All these BSS algorithms are contained in the EEGLAB,${ }^{8}$ FastICA, ${ }^{16}$ or $\mathrm{ICALAB}^{5}$ toolboxes for Matlab.

AMUSE $^{37}$ and SOBI $^{3}$ are time-structure-based methods, also known as second-order statistics (SOS) BSS. They assume that the sources have no spatialtemporal correlations. ${ }^{22}$ Thus, these techniques diagonalize a set of cross-covariance matrices of $\mathbf{x}(t)$. AMUSE only considers two time delays $(\tau=0$ and $\tau=1$ samples), ${ }^{37}$ whereas SOBI uses iterative procedures to simultaneously diagonalize multiple temporal lags. ${ }^{3}$ SOBI is applied with 50 consecutive time lags, from $\tau=1$ sample $(\tau \approx 0.006$ s) to $\tau=50$ samples $(\tau \approx$ $0.295 \mathrm{~s}$ ) because this set of delays covers a wide time interval without extending beyond the support of the average autocorrelation function of the MEG recordings. ${ }^{10}$

On the other hand, JADE, ${ }^{4}$ eInfomax, ${ }^{26}$ and FastICA $^{20}$ rely on higher-order statistics (HOS) such as negentropy and kurtosis. They look for nonGaussian sources assuming that $\mathbf{x}(t)$ are observations of random variables where temporal order is irrelevant. ${ }^{20,22}$ FastICA is applied with the non-linearity $\tanh (\cdot)$ and the deflationary approach. ${ }^{20}$ This function is selected for being a good general-purpose function. ${ }^{20}$ eInfomax is used to estimate both sub- and superGaussian sources. ${ }^{26}$ The number of each type of components is automatically determined. ${ }^{26}$ JADE has no input parameters. ${ }^{4,20,40}$

The previous algorithms decompose the signals into a set of sources. An alternative is to guide the BSS so that only the components close to a signal of interest - reference: $r(t)$-are extracted. This is called cBSS and requires a fair estimate of the activity of interest. ${ }^{22}$ In this case, the cBSS removes the need for identification and labeling of the components. ${ }^{21}$ The cBSS algorithms convert the constrained problem to an unconstrained one by introducing a regularization 
parameter in the decomposition. ${ }^{19,29}$ Thus, one obtains a component that is statistically independent of other sources and closest to $r(t){ }^{22} r(t)$ does not need to be perfectly identical to the source of interest, but it should be similar enough to drive the algorithm into the direction of the desired component. ${ }^{22}$ In this study, the cBSS implementation by Huang and Mi is used. ${ }^{19}$

\section{Artifact Detection Metrics}

Some widespread metrics to recognize the CA, OA, and PLN are compiled in this study. We do not pretend to provide a full picture of all proposed metrics, but to offer a broad vision of the types of approaches commonly employed in the Literature.

When a probability distribution is computed, the number of bins of the histogram is set to the number of data samples divided by five. ${ }^{9}$ Additionally, a 'segment' approach is taken to compute the artifact detection metrics based on statistical parameters. This procedure consists of dividing the components into non-overlapping segments of 1 s (169 data samples) and the metric is separately computed for each segment. $^{2,7,30}$ In case a certain fraction of the segments of a component exceeds a predefined threshold, the component is marked for removal. ${ }^{2,7,30}$ The distributions derived from the 'segment' approach were normalized to zero mean and SD equal to 1 with respect to all components extracted with the same BSS algorithm and epoch length. ${ }^{2,7,30} \mathrm{~A}$ component is marked as artifact if $30 \%$ or more of its segments exceeds a value of \pm 2.0 after the normalization. ${ }^{7}$ This criterion is more restrictive than the commonly used one that consists of rejecting a BSS source if $20 \%$ or more of its segments exceeded a value of $\pm 1.64,{ }^{2}$ and it aims at minimizing the false positives in the artifact detection. ${ }^{7}$

\section{Cardiac Artifact}

The following metrics are used in the detection of cardiac components.

Skewness (Skew): Skewness (Skew) is used to detect CAs isolated in BSS components. ${ }^{7,12,35}$ Skew is the normalized third central moment of the amplitude distribution of the signal. Only if the amplitude distribution is symmetrical, Skew is zero. ${ }^{12}$ Thus, large abs(Skew) values, where $\operatorname{abs}(\cdot)$ denotes absolute value, are associated with asymmetric components, which may be due to cardiac activity. ${ }^{12}$

Variance of the Scalp Distribution (VarSc): The scalp distributions associated with CA may have small variance. ${ }^{35}$ Hence, this metric is computed as the variance of the column vectors of $\hat{\mathbf{A}}$ associated with each component. ${ }^{35}$ Due to the fact that this metric intends to detect components whose scalp distribution has extremely low variance and that the CA always appears in the recordings, ${ }^{12}$ the component with the minimum value of VarSc for each signal epoch is marked for rejection.

Kurtosis Excess ( $\mathrm{KrE}$ ): Kurtosis has been used in several studies to recognize diverse artifacts, including $\mathrm{CA}$ and $\mathrm{OA} .{ }^{2,7,12,30}$ Kurtosis excess ( $\mathrm{KrE}$ ) is derived from the normalized fourth central moment of the amplitude distribution. $\mathrm{KrE}$ is negative for subGaussian amplitude distributions. Alternatively, $\mathrm{KrE}$ is positive for super-Gaussian ones. ${ }^{2,9,12}$ Extreme values of $\mathrm{KrE}$ are related to abnormal components. $\mathrm{KrE}$ is tested in the rejection of all kind of artifacts considered in this study.

Shannon Entropy (ShEn): Entropy measures the disorder, or irregularity, in a signal. Higher entropy values correspond to more unstructured signals. On the other hand, small entropy values correspond to components whose amplitude distributions are contained in few limited intervals with high probabilities, as it happens in certain types of artifacts. ${ }^{2,7}$ Thus, the well-known Shannon entropy ( $\mathrm{ShEn}$ ) is used to mark BSS components with all types of artifacts considered in this study.

Rényi Entropy (RéEn): ShEn can be replaced with Rényi entropy (RéEn) in the detection of artifactual components. ${ }^{30}$ A quadratic entropy index $(q=2)$ is used to put equal emphasis on all data points regardless of their probability density. ${ }^{30}$ This metric is tested in the detection of CA, OA, and PLN.

Approximate Entropy (ApEn): Another entropic measure able to detect artifactual components in MEG activity is approximate entropy (ApEn). ${ }^{31} \mathrm{ApEn}$ quantifies the regularity of a sequence and is expected to be small for non-cerebral biological signals, such as $\mathrm{CA}$ and $\mathrm{OA}{ }^{31} \mathrm{~A}$ run length and a tolerance window must be specified to compute it. In this case, ApEn is computed with a run length of 2 and a tolerance window of 0.2 times the SD of the signal. ${ }^{31}$ ApEn is tested to detect CA, OA, and PLN.

Skew, KrE, ShEn, RéEn, and ApEn are applied to the components following the previously introduced 'segment approach'.

Constrained Blind Extraction of the CA: A cBSS approach is also taken to remove the CA from the MEGs. ${ }^{21}$ The cBSS extracts components characterized by having minimal dependence and being similar to a reference signal. ${ }^{19,21}$ The reference- $r(t)$-for the cBSS is computed considering that MEG background activity was acquired. The background brain activity spreads over a relatively broad range of frequencies. On the other hand, the CA introduces synchronous peaks in all MEG channels. Thus, the activity recorded at each time instance at all 148 MEG channels is averaged. The resulting signal resembles an electrocardiogram 
(ECG) because this averaging process emphasizes the CA that contaminates all channels and tends to cancel out the brain activity. ${ }^{12}$ Yet, the average of all MEG channels is not a pure cardiac signal since some other activities may also appear in it. Fortunately, the reference for the cBSS only needs to capture the main temporal features of the signal of interest. ${ }^{21}$ Hence, the actual $r(t)$ fed to the cBSS algorithm is created as a binary signal with l's where the R-peaks of the cardiac activity appear in the average signal and 0's elsewhere. Once this binary reference signal has been obtained, it is used with a cBSS algorithm to extract a component that accounts for the CA. Then, this component is subtracted from the recordings. ${ }^{19,21}$

\section{Ocular Artifact}

The reduction in the OA is also quantified. The ocular components share some characteristics with the CA (e.g., extreme values of kurtosis or entropy). Thus, $\mathrm{KrE}$, ShEn, RéEn, and ApEn are also used to mark OAs. ${ }^{2,7,30,31}$ Additionally, two metrics designed ad hoc for the detection of the $\mathrm{OA}$ are tested. They are detailed below.

Power near the eyes $\left(P_{\text {eyes }}\right)$ : The power of the $\mathrm{OA}$ is mainly gathered near the eyes. Hence, the scalp distribution of the components is used in the detection of this artifact. ${ }^{12,14,28}$ We compute a metric (Power near the eyes, $\left.P_{\text {eyes }}\right)$ as the fraction of the power located on the 13 frontal peripheral channels nearer to the eyes. ${ }^{12}$ We intend to detect components whose $P_{\text {eyes }}$ is extremely high. Yet, not all signal epoch are contaminated with $\mathrm{OA}$. Therefore, in order to minimize the removal of non-artifactual activity, the values of $P_{\text {eyes }}$ are normalized to zero mean and $\mathrm{SD}=1$ with respect to all components derived from the same BSS method and epoch length. ${ }^{7}$ Then, following Ref. 7 we set the detection threshold to +3.5 as this level is applied to a statistic derived from the full signal segment and we expect the OAs to be characterized with very high $P_{\text {eyes }}$ values.

Power in Low Frequencies $\left(P_{l f}\right)$ : The energy of the OA appears in low frequencies. ${ }^{12,14,24}$ Thus, we compute the fraction of the power spectral density (PSD) that each BSS component has from 0.5 to $2.5 \mathrm{~Hz}$ (Power in Low Frequencies, $P_{\mathrm{If}}$ ). ${ }^{12}$ Similarly to $P_{\text {eyes}}$, the values of $P_{\text {If }}$ are normalized to zero mean and SD $=1$ with respect to all the relevant components. Then, the detection threshold is set to +3.5 to minimize the amount of lost brain activity. ${ }^{\text {? }}$

\section{Power Line Noise}

Due to the expected rhythmic nature of the PLN, its amplitude distribution is different from a typical brain signal. Hence, KrE, ShEn, RéEn, and ApEn are employed in its detection. ${ }^{31}$ In addition, the following metric is tested.

Power at the Line Frequency $\left(P_{50}\right)$ : If a component completely isolates PLN, its spectrum will be centered at the power line frequency $\left(50 \mathrm{~Hz}\right.$ in this case). ${ }^{12,25,27}$ Hence, we calculate a spectral metric $\left(P_{50}\right)$ that measures the fraction of the PSD contained from $49.5 \mathrm{~Hz}$ to $50.5 \mathrm{~Hz}$ for each component. ${ }^{12}$ Large values of $P_{50}$ are due to BSS components that have most of their energy round $50 \mathrm{~Hz}$. For each BSS algorithm and epoch length, all $P_{50}$ values are normalized to zero mean and SD 1 and a threshold of +3.5 is applied, following a similar reasoning to that of $P_{\text {eyes }}$.

\section{Artifact Removal Evaluation}

It is of paramount importance to quantify the reduction in the artifactual activity in real brain signals without time-consuming visual inspections. The evaluation criteria applied to achieve this goal are described in the following lines. By comparing the values of these criteria before and after the BSS-based artifact removal, it is possible to evaluate the amount of removed artifactual activity.

\section{Cardiac Artifact}

In order to assess the CA removal, we detect the QRS-complexes in the average of all 148 MEG channels in the same way as it was done to compute the reference for the cBSS. ${ }^{12}$ Therefore, the R-peaks are located in the average signal before the artifact rejection process and an average QRS-complex is estimated before and after each artifact removal. ${ }^{12}$ Then, the importance of the CA is characterized with the Peakto-Peak Amplitude $\left(A_{\mathrm{pp}}\right)^{12,31}$ and the root mean square (RMS) value ${ }^{7}$ of the mean QRS complex for each subject.

\section{Ocular Artifact}

The evaluation of the OA removal is more complex than the cases of the CA and PLN. The ocular activity does not appear in all MEG epochs. However, the effects of the BSS artifact rejection procedure on the MEG signals can still be quantified. For every signal epoch, the average PSD is computed at the subset of 18 MEG channels located over the anterior part of the head and close to the eyes and at the remaining set of 130 channels. It is important to note that the first subset of channels is not equal to that used in the metric $P_{\text {eyes }}$ to avoid biasing the results. The subtraction of those two PSDs computed over completely disjoint sets of channels shows the difference between the activity recorded over those two areas as a function 
of frequency. Hence, one calculates the difference between the PSDs from 0.5 to $6.5 \mathrm{~Hz}$ as most of the OA energy is located at low frequencies. ${ }^{34}$ Additionally, an amplitude threshold is set in the MEG recordings to count the number of peaks in the signals whose amplitude is above $\pm 2 \mathrm{pT}$ before and after the artifact removal. This measurement is computed as an indication of the suppression of peaks in the recordings. ${ }^{31}$ The threshold is set at $\pm 2 \mathrm{pT}$ as a good tradeoff between avoiding the detection of CA R-waves and the number of detected peaks in the MEGs.

\section{Power Line Noise}

The reduction of the PLN is assessed by calculating the mean of the PSD and normalized PSD $\left(\mathrm{PSD}_{n}\right)$ of the MEGs before and after the artifact rejection and inspecting the amount of power contained between 49 and $51 \mathrm{~Hz}^{12}$

\section{RESULTS}

This section contains the results of the objective evaluation of the artifact removal in real MEG epochs. Due to the high number of combinations of BSS algorithm, epoch length, artifact detection metric, and evaluation criteria, only a summary of the results is presented here. We have focused on the most remarkable cases trying to illustrate large and small reductions in the artifacts. For the sake of completeness, all numerical results are compiled into the tables presented in Appendix (Supplementary material), which the interested reader is referred to.

First of all, it was necessary to measure the level of the artifacts in the original MEG recordings. This enabled us to have reference values for an objective assessment of the artifact removal. ${ }^{12}$ Therefore, the metrics described in "Artifact Removal Evaluation" section were applied to the MEG signals before any kind of BSS processing. The presence of the CA was evaluated considering the amplitude and power of the average QRS complex found in the MEGs. R-peaks were located in the average of all 148 MEG channels, ${ }^{12}$ and $A_{\mathrm{pp}}$ and RMS values were computed for the original MEGs. Figure 1 depicts the average QRS complex computed from all subjects for epochs of $10 \mathrm{~s}$. As it can be seen in Fig. 1, the CA is considerably strong in the MEG recordings and, on average, introduces well-defined peaks in the MEG recordings. As for the OAs, an amplitude threshold of $\pm 2 \mathrm{pT}$ was set to count the number of these 'high-amplitude' peaks in the recordings. ${ }^{31}$ An average PSD 'discrepancy' measure was also computed to consider the increase in the power of low frequencies near the eyes due to the OA. Figure 2 illustrates this spectral measure for epochs of
$20 \mathrm{~s}$ and it shows the increase in low frequency power near the eyes. Finally, the assessment of the PLN power in the MEGs was performed by calculating the power between $49 \mathrm{~Hz}$ and $51 \mathrm{~Hz}$ in the PSD and $\mathrm{PSD}_{n}$. An illustration of this part of the spectra is shown for epochs of $60 \mathrm{~s}$ in Fig. 3, which clearly reveals the contamination with a harmonic at the power line frequency. Other epoch lengths than those represented in Figs. 1, 2, and 3 have almost identical levels of $\mathrm{CA}$, $\mathrm{OA}$, and PLN contamination.

After the computation of the values that indicated the average artifact level in the original MEGs, the actual process of artifact removal started. The preprocessing and dimension reduction techniques indicated in "Preprocessing and Model Order Selection" section were applied to MEG epochs of 10, 20, 40,60, and $90 \mathrm{~s}$. The optimum number of inner components (l) was estimated with the MDL. The results of the

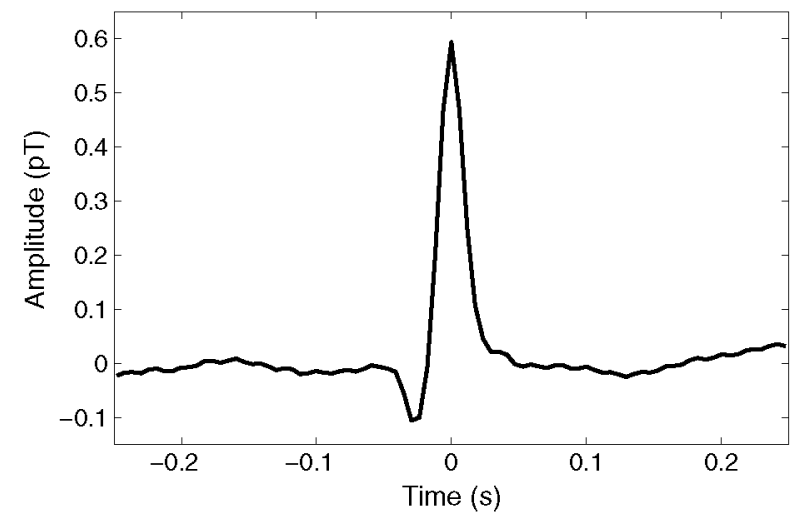

FIGURE 1. Average QRS complex in the original MEG recordings for epochs of $10 \mathrm{~s}$. The QRS complex becomes apparent after averaging the MEG channels and illustrates the strength of this artifact in the signals.

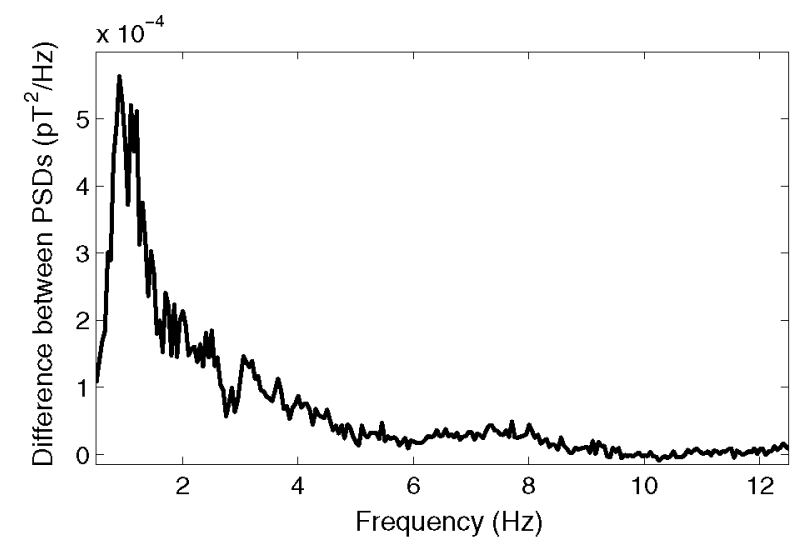

FIGURE 2. Median of the difference between the PSDs for the two subsets of MEG channels used to compare the impact of OAs in the MEG recordings for epochs of $20 \mathrm{~s}$. The large positive values of this spectral metric, especially below $4 \mathbf{H z}$, highlight the presence of increased low frequency activity at the channels near the eyes in the original MEG recordings. 
preprocessing stage are reported elsewhere. ${ }^{10}$ Then, the BSS algorithms described above were used to decompose the recordings. In addition, a cBSS algorithm was used to extract the cardiac contamination from the recordings and then cancel it out by subtracting its projection. $^{21}$

Once the decomposition had been performed, the next step was to apply the artifact detection criteria specified in "Artifact Detection Metrics" section. This enabled us to automatically label the sources with abnormal values of those metrics as artifacts. Of note is that $\mathrm{KrE}, \mathrm{ShEn}$, RéEn, and ApEn were used to detect all types of artifacts included in this study. Finally, a reconstruction of the MEGs was calculated by means of Eq. (3) and the measures to evaluate the amount of artifacts were applied to these reconstructed
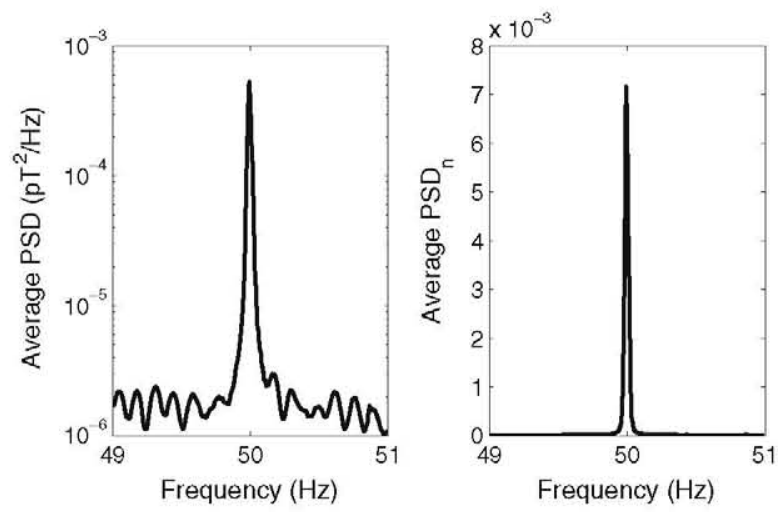

FIGURE 3. Average spectra between 49 and $51 \mathrm{~Hz}$ computed from epochs of $60 \mathrm{~s}$. It can be seen that the PLN introduces a clearly visible harmonic at $50 \mathrm{~Hz}$ in the original MEG recordings.

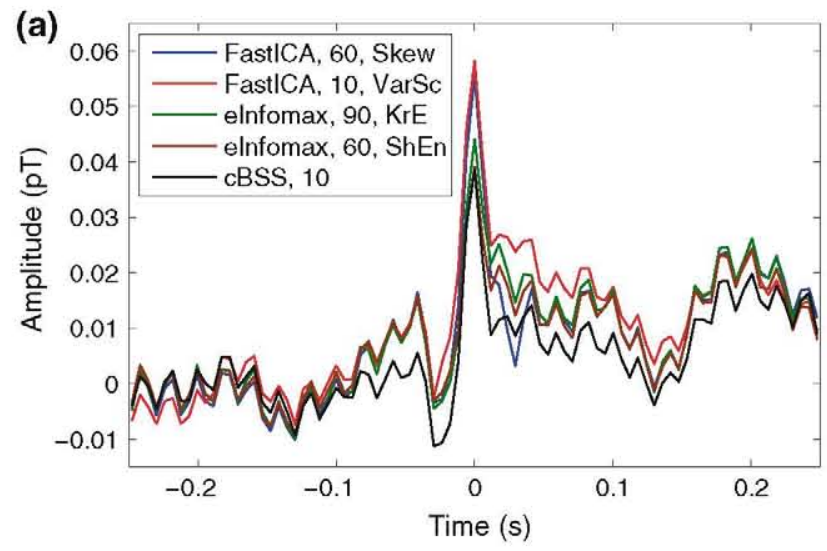

signals. It is important to bear in mind the results computed for the original recordings and Figs. 1, 2, and 3 to assess how much artifactual activity was removed in each case.

For the assessment of the CA removal, the $A_{\mathrm{pp}}$ and RMS values were calculated from the locations where the $\mathrm{R}$-peaks appeared in the raw recordings (see "Cardiac Artifact" section). These numeric results, together with those for the OA and PLN, are given in Appendix (Supplementary material). Moreover, a few representative cases of larger and smaller reductions of the CA are illustrated in Fig. 4, which depicts the remainder of the QRS complex after the artifact removal. Large reductions in the CA were achieved with several combinations of BSS algorithm, epoch length, and artifact detection criteria. These include the use of eInfomax with $\mathrm{KrE}$ on epochs of $90 \mathrm{~s}$, FastICA with Skew on epochs of $60 \mathrm{~s}$ or a cBSS approach on epochs of $10 \mathrm{~s}$. The reduction in the power of the CA is apparent with this techniques, since the maximum amplitude of the CA decreased in approximately one order of magnitude thanks to the BSS artifact removal. On the other hand, SOS-BSS techniques tended to offer smaller reductions in the CA. It must be noted that the amplitude ranges of Fig. 1 and the subplots of Fig. 4 are different.

Regarding the OA removal, two criteria were proposed in "Ocular Artifact" section to evaluate how the BSS modified the MEG signals and the influence of the OAs on them. The first one was based on counting the number of peaks exceeding $\pm 2 \mathrm{pT}$. These numeric results are given in Appendix (Supplementary material). The second criterion considered the difference between two subsets of channels in a particular spectral band.

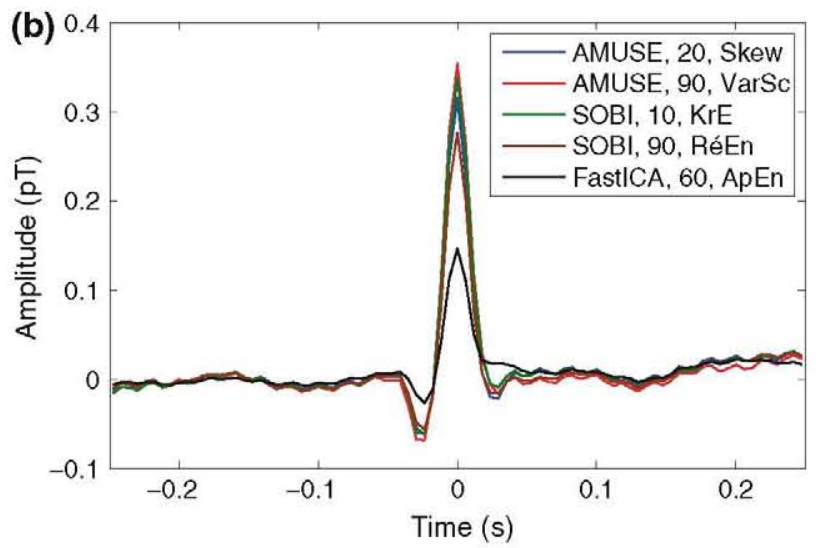

FIGURE 4. Examples of larger (a) and smaller (b) reductions in the average QRS complex after the BSS-based CA removal. (a) The combinations of FastICA and Skew on epochs of $60 \mathrm{~s}$, FastICA and VarSc on epochs of $10 \mathrm{~s}$, elnfomax and KrE on epochs of $90 \mathrm{~s}$, elnfomax and ShEn on epochs of $60 \mathrm{~s}$, and cBSS on epochs of $10 \mathrm{~s}$ largely reduced the CA. On the other hand, examples of smaller CA removal include AMUSE and Skew on epochs of $20 \mathrm{~s}$, AMUSE and VarSc on epochs of $90 \mathrm{~s}$, SOBI and KrE on epochs of $10 \mathrm{~s}$, SOBI and RéEn on epochs of $90 \mathrm{~s}$, and, to a lesser extent, FastICA and ApEn on epochs of $60 \mathrm{~s}$ (b). The reduction in the CA is obvious comparing this signals with that of Fig. 1, as the maximum amplitude decreased from 0.6 to $0.06 \mathrm{pT}$. 
(a)

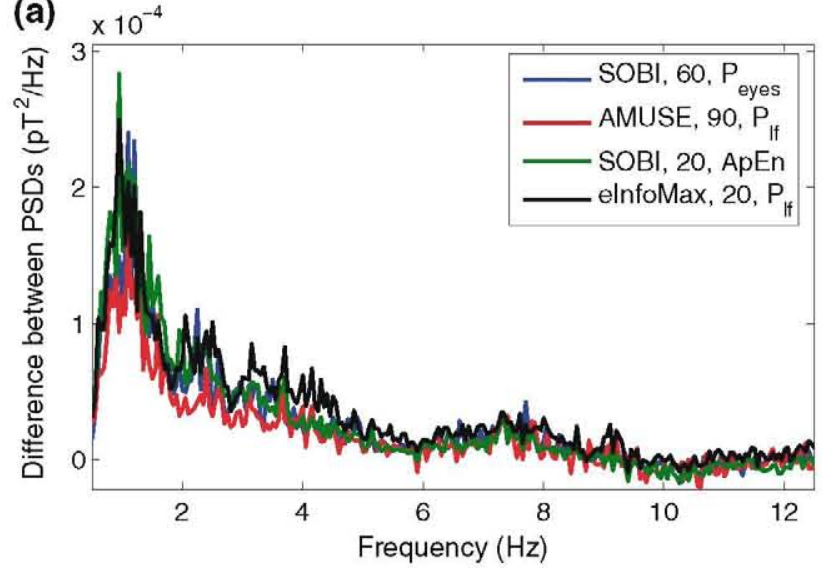

(b)

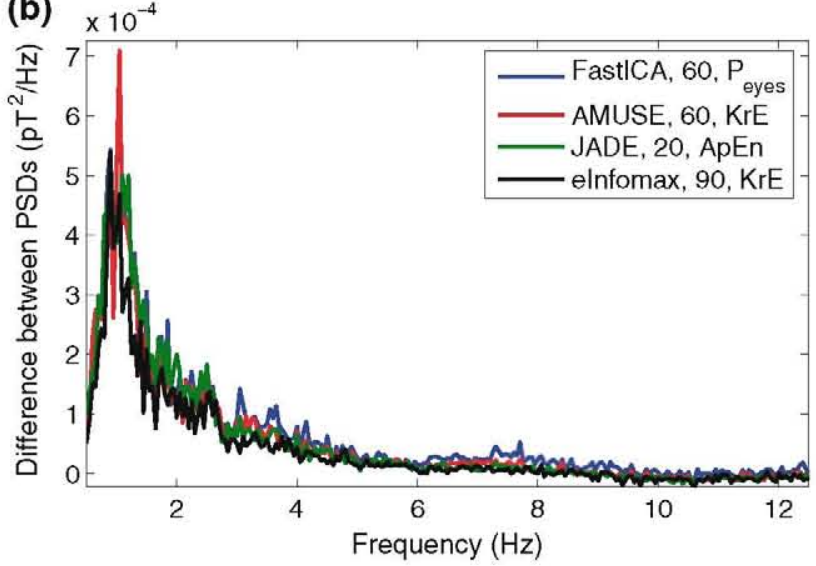

FIGURE 5. Examples of larger (a) and smaller (b) reductions in the median of the spectral discrepancy measure near the eyes after the BSS-based OA removal. (a) Some of the combinations that provided large reductions in the low frequencies: SOBI and $P_{\text {eyes }}$ on epochs of $60 \mathrm{~s}, \mathrm{SOBI}$ and ApEn on epochs of $20 \mathrm{~s}$, AMUSE and $\boldsymbol{P}_{\text {If }}$ on epochs of $90 \mathrm{~s}$, and elnfomax and $\boldsymbol{P}_{\text {If }}$ on epochs of 20 s. (b) Some of the combinations which offered small reductions in the discrepancy, namely FastiCA and $P_{\text {eyes }}$ on epochs of $60 \mathrm{~s}$, AMUSE and KrE on epochs of 60 s, JADE and ApEn on 20 s, and elnfomax and KrE on epochs of 90 s. In comparison with Fig. 2, the spectral discrepancy decreased, at least, to one half in the best cases.
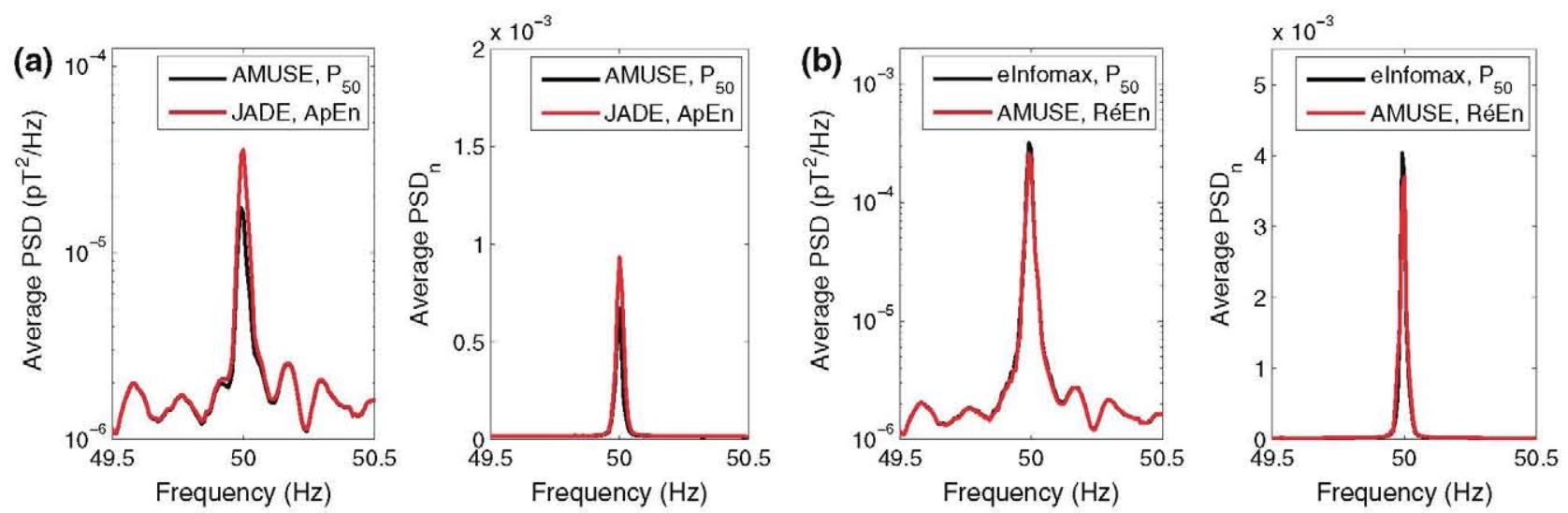

FIGURE 6. Examples of larger (a) and smaller (b) reductions in the average spectra centered at the PLN frequency after the BSSbased PLN removal. Panel (a) represents clear reductions in power at $50 \mathrm{~Hz}$. These were achieved with AMUSE and $P_{50}$ on epochs of $60 \mathrm{~s}$ and JADE and ApEn on epochs of $60 \mathrm{~s}$. Panel (b) exemplifies less successful PLN removal strategies: elnfomax and $P_{50}$ on epochs of $60 \mathrm{~s}$ and AMUSE and RéEn on epochs of $60 \mathrm{~s}$. By contrasting Fig. 6 with Fig. 3, it is clear that the best combinations decreased the power at $50 \mathrm{~Hz}$ by more of one order of magnitude without distorting the spectrum around this frequency.

This spectral difference is illustrated in Fig. 5 for a few representative cases of larger and smaller reductions in low frequency power near the eyes. The spectral discrepancy was decreased with approaches like SOBI and $P_{\text {eyes }}$ for epochs of $60 \mathrm{~s}$, SOBI and ApEn for epochs of $20 \mathrm{~s}$ or AMUSE and $P_{\mathrm{lf}}$ for epochs of $90 \mathrm{~s}$. In these cases, the power of this spectral discrepancy decreased to about $50 \%$ due to the use of the BSS approaches, as it can be seen in comparison with Fig. 2. Of note is that the scales of these two figures differ. On the other hand, Fig. 5 also illustrates cases for which the OA removal strategies did not decrease the amount of power in low frequencies near the eyes, such as FastICA and $P_{\text {eyes }}$ on epochs of $60 \mathrm{~s}$, AMUSE and $\mathrm{KrE}$ on epochs of $60 \mathrm{~s}$ or JADE and ApEn on $20 \mathrm{~s}$.
Finally, the effectiveness of the reduction in the PLN with a BSS-based artifact removal was measured in the frequency domain. We considered the power of the PSD and $\operatorname{PSD}_{n}$ around $50 \mathrm{~Hz}$, as indicated in "Power Line Noise" section Fig. 6 offers visual information about the reduction in the PLN for four cases. Two of them show a clear decrease in the harmonic at $50 \mathrm{~Hz}$ (AMUSE and $P_{50}$, and JADE and ApEn, in both cases for epochs of $60 \mathrm{~s}$ ), whereas the other two are representative of smaller diminishings: eInfomax and $P_{50}$, and AMUSE and RéEn, also for epochs of 60 s. A comparison of Fig. 3 with Fig. 6, which were plotted with different amplitude scales, reveals a reduction slightly larger than one order of magnitude in the power of harmonic at $50 \mathrm{~Hz}$ with the best 
BSS-based PLN removal procedures. Furthermore, this procedures avoid the distortion that spectral filtering could have introduced in the signals.

\section{DISCUSSION AND CONCLUSIONS}

We compared widespread BSS algorithms and artifact detection metrics in terms of their ability to reduce the CA, OA, and PLN in real MEG recordings from 26 elderly subjects for several epoch lengths. Our aim was to objectively quantify the artifact removal without having to rely on visual inspections of the data, which are subjective and time-consuming. The results showed a clear reduction in the amount of $\mathrm{CA}$ and PLN in the signals thanks to the BSS artifact removal, whereas the OA results also pointed to a reduction of this kind of artifact but they were more difficult to assess.

The BSS techniques were applied after a preprocessing and model order selection. ${ }^{10,12}$ This preprocessing was also used before the cBSS to ensure that all decomposition or extraction methods were applied to the same input data. After the BSS, several artifact detection metrics and criteria helped to identify the potential artifacts.

None of the artifact removal strategies tested in this study relies on the recording of auxiliary signals, such as the ECG and EOG. This is a remarkable advantage when recording brain activity from elderly people, who may suffer from some level of cognitive impairment, infants, and non-collaborative subjects in general. On the other hand, the lack of these auxiliary signals hinders the evaluation of the artifact removal. For instance, if the EOG had been acquired, it would have been possible to inspect the MEGs around the OAs captured in that signal. ${ }^{7}$ This issue is less problematic in the case of the CA because background MEG activity was analyzed. Thus, the average of all MEG channels tended to emphasize this artifact. ${ }^{12}$ Therefore, the fact that auxiliary signals were not included in the analysis makes the evaluation of the artifact removal (especially the OA) more difficult and limits the results. However, this makes our findings directly applicable to situations where the auxiliary signals either can or cannot be recorded. Moreover, it should be borne in mind that we aimed at performing a completely automatic quantification of the artifact removal, without visual inspections of the signals.

Several publications have tried to remove the CA in MEG data with diverse approaches, including higher order statistics and entropy measures, ${ }^{2,7,31}$ correlation, ${ }^{2}$ phase information, ${ }^{7}$ variability of the scalp maps, ${ }^{35}$ and asymmetry. ${ }^{12,35}$ Considering these studies, we selected a few metrics to label the CA. Among them, Skew and VarSc were only applied to the detection of the CA, while KrE, ShEn, RéEn, and ApEn were tested on the detection and removal of all types of artifacts on the basis of previous studies. ${ }^{2,7,30,31}$ On the other hand, cBSS can extract artifactual activity if a suitable reference is available. ${ }^{21}$

The OA removal with BSS has received considerable attention in $\mathrm{MEG}^{2,7,12,32}$ and, particularly, EEG. ${ }^{24,25,27,28,33} 36$ The metrics used to detect the OA have been based on topographic information, ${ }^{12,28,32}$ correlation, ${ }^{2,24}$ thresholding, ${ }^{36}$ and higher-order statistics. $^{7}$ The diverse types of signals and evaluation methodologies make the direct comparison of the results difficult. Hence, we compared straightforward criteria $\left(P_{\text {eyes }}, P_{\mathrm{lf}}, \mathrm{KrE}\right.$, ShEn, RéEn, and ApEn) in the $\mathrm{OA}$ detection. $P_{\text {eyes }}$ and $P_{\mathrm{If}}$ identified less components as artifacts, although it must be noted that the other criteria were supposed to pinpoint all types of contamination. The MEGs were recorded while the subjects had their eyes closed. Hence, the number of blinks and fast eye movements could be reduced but the power in the $\alpha$ band could have increased as a result. Moreover, the lack of a visual reference might increase the low-frequency eye movements. ${ }^{6}$ Despite the fact that this might induce artifacts in the recordings, the metric $P_{\mathrm{lf}}$, which detects low frequencies, might deal with this situation. Nonetheless, the application of this metric to open-eyes settings may not be straightforward. ${ }^{12}$

The reduction of the PLN is typically done by spectral filtering but another approach is needed when the spectral band of interest overlaps with the power line frequency. ${ }^{25}$ The detection of the PLN components was done by taking into account the power around $50 \mathrm{~Hz}{ }^{12}$ Similar criteria have been introduced, ${ }^{27}$ although the PLN removal was not measured individually.

Most artifact detection criteria were applied to 'segments' of BSS components. , $7,17,30^{\text {In order to }}$ minimize the amount of removed brain components, we only marked a BSS source as an artifact if $30 \%$ or more of its segments exceeded a threshold value of \pm 2.0 once the detection metric had been normalized to zero mean and $\mathrm{SD}=1 .^{7}$ This criterion decreases the amount of removed artifactual activity but it safeguards the brain activity. ${ }^{7}$ Furthermore, this type of criteria is appropriate to label cases for very unbalanced data. The detection metrics aiming at labeling all artifacts marked less components for removal than the combination of the metrics designed for a specific kind of contamination. This suggests that the former, by trying to recognize different artifacts, may lose some sensitivity to the undesired sources.

On the basis of the results, Table 1 indicates a few of the best combinations of BSS algorithm, epoch length, 
and artifact detection metric that offered the largest artifact reduction. These data are expressed as the ratio of the values of the corresponding metric after and

TABLE 1. Summary of the changes (expressed as ratios) in the metrics to assess the artifact removal for some of the best combinations of BSS algorithm, epoch length, and artifact detection criterion.

\begin{tabular}{|c|c|c|c|c|}
\hline \multicolumn{5}{|c|}{ Ratios of the metrics evaluating the $\mathrm{CA}$} \\
\hline Metric & Length (s) & BSS method & $A_{\mathrm{pp}}$ & RMS \\
\hline- & 10 & cBSS & 0.1335 & 0.1963 \\
\hline Skew & 60 & FastlCA & 0.1626 & 0.2437 \\
\hline VarSc & 10 & FastlCA & 0.1572 & 0.2513 \\
\hline KrE & 90 & elnfomax & 0.1354 & 0.2258 \\
\hline ShEn & 60 & elnfomax & 0.1238 & 0.2132 \\
\hline \multicolumn{5}{|c|}{ Ratios of the metrics evaluating the $\mathrm{OA}$} \\
\hline Metric & Length $(s)$ & $\begin{array}{c}\text { BSS } \\
\text { method }\end{array}$ & $\begin{array}{l}\text { Number } \\
\text { of peaks }\end{array}$ & $\begin{array}{c}\text { Spectral } \\
\text { discrepancy }\end{array}$ \\
\hline$P_{\text {eyes }}$ & 60 & SOBI & 0.6158 & 0.4137 \\
\hline$P_{\mathrm{If}}$ & 90 & AMUSE & 0.1589 & 0.4357 \\
\hline ApEn & 20 & SOB & 0.2848 & 0.4011 \\
\hline$P_{\text {If }}$ & 20 & elnfomax & 0.5858 & 0.5263 \\
\hline \multicolumn{5}{|c|}{ Ratios of the power from 49.5 to $50.5 \mathrm{~Hz}$ to evaluate the PLN } \\
\hline Metric & Length $(s)$ & $\begin{array}{c}\text { BSS } \\
\text { method }\end{array}$ & $\begin{array}{l}\text { Total } \\
\text { power }\end{array}$ & $\begin{array}{c}\text { Normalized } \\
\text { power }\end{array}$ \\
\hline$P_{50}$ & 60 & AMUSE & 0.2039 & 0.1850 \\
\hline ApEn & 60 & JADE & 0.2551 & 0.3178 \\
\hline
\end{tabular}

Lower ratios indicate the removal of larger amounts of artifacts. before the artifact removal. The list shown in Table 1 accounts for some of the combinations illustrated in Figs. 4, 5, and 6. Nonetheless, it is not exhaustive as slight modifications (i.e., shorter or longer epoch lengths or using RéEn instead of ShEn) offer similar levels of artifact reduction, something that suggests high consistency in the performance of the BSS-based artifact removal. Nonetheless, Table 1 provides an indication of which specific combinations of BSS algorithm, epoch length, and artifact detection metric are best suited for CA, OA, and PLN with respect to the applied criteria.

Table 1 suggests that metrics specifically designed for the detection of each type of artifact might be superior to criteria used to deal with all kinds of artifacts. Yet, in situations where all forms of artifacts are expected, it may be desirable to apply just one procedure able to reduce all artifacts. In order to further clarify the results and to address this issue, Table 2 presents a summary of the performance achieved with various BSS algorithms and artifact detection metrics. Considering that, overall, the effect of the epoch length in the performance of the artifact removal was far less important than that of the interaction between the BSS algorithm and the artifact detection metric, we averaged the performance of all combinations tested in this study over all epoch lengths (results shown in Appendix, see Supplementary material). Then, we further summarized the results by showing in Table 2 only the results for one HOS- and one SOS-BSS algorithm for every 'general' detection criterion. For the specific artifact labeling methods, only the results of the BSS

TABLE 2. Summary of the changes, expressed as ratios and averaged over all five epoch lengths, in the metrics used to assess the artifact removal.

\begin{tabular}{|c|c|c|c|c|c|c|c|}
\hline \multirow[b]{2}{*}{ Detec. crit. } & \multirow[b]{2}{*}{ BSS alg. } & \multicolumn{2}{|c|}{$\mathrm{CA}$} & \multicolumn{2}{|c|}{$O A$} & \multicolumn{2}{|c|}{ PLN } \\
\hline & & $A_{\mathrm{pp}}$ & RMS & \# peaks & Discrepancy & PSD power & $\mathrm{PSD}_{n}$ power \\
\hline $\mathrm{KrE}$ & SOBI & 0.5611 & 0.5511 & 0.4827 & 0.8653 & 0.9997 & 1.2025 \\
\hline $\mathrm{KrE}$ & FastlCA & 0.1451 & 0.2419 & 0.4899 & 0.8793 & 0.9963 & 1.3152 \\
\hline ShEn & SOBI & 0.5299 & 0.5258 & 0.4005 & 0.7382 & 0.9994 & 1.2165 \\
\hline ShEn & FastlCA & 0.1382 & 0.2365 & 0.4587 & 0.8264 & 0.9960 & 1.3188 \\
\hline RéEn & SOBI & 0.5340 & 0.5284 & 0.3384 & 0.6498 & 0.8630 & 0.9948 \\
\hline RéEn & FastlCA & 0.1401 & 0.2374 & 0.4514 & 0.8072 & 0.9962 & 1.3186 \\
\hline ApEn & SOBI & 0.7801 & 0.6833 & 0.3597 & 0.4918 & 0.3701 & 0.5119 \\
\hline \multirow[t]{2}{*}{ ApEn } & elnfomax & 0.2720 & 0.3226 & 0.3153 & 0.6966 & 0.5995 & 0.6802 \\
\hline & cBSS & 0.1618 & 0.2209 & - & - & - & - \\
\hline Skew & FastlCA & 0.1676 & 0.2572 & - & - & - & - \\
\hline VarSc & FastlCA & 0.2065 & 0.3124 & - & - & - & - \\
\hline$P_{\text {eyes }}$ & SOBI & - & - & 0.6841 & 0.5739 & - & - \\
\hline$P_{\mathrm{If}}$ & AMUSE & - & - & 0.3642 & 0.4374 & - & - \\
\hline$P_{50}$ & AMUSE & - & - & - & - & 0.2094 & 0.1935 \\
\hline
\end{tabular}

Lower ratios indicate the removal of larger amounts of artifacts. Only the results of one SOS- and one HOS-BSS are specified for each of the artifact detection techniques ('Detect. crit.') that deal with all kinds of artifacts. For a comprehensive list of the results, the reader is referred to the Appendix in Supplementary material. 
algorithm with the highest artifact removal are given. The algorithms were selected as those that achieved the greatest performance in the highest number of cases. The selection of one HOS- and one SOS-BSS technique was motivated by the fact that the decomposition calculated with AMUSE and SOBI tend to be more similar than those of JADE, eInfomax, and FastICA, and vice versa. ${ }^{10}$

In general, FastICA seems to be the HOS-BSS method with the highest removal ratios for all forms of artifacts. This agrees with a previous study on the consistency of the BSS of MEG signals. ${ }^{10}$ On the other hand, the results suggests a slight superiority of SOBI over AMUSE. Table 2 also confirms that the specifically designed detection criteria may provide larger artifact removal than the 'general' ones. Furthermore, the results indicate that, most often, HOS-BSS algorithms are able to remove large fractions of the CA. The cBSS procedure is relatively simple and can reject most of the CA as well. The $A_{\mathrm{pp}}$ and RMS values decreased from about 0.750 and 0.120 to around 0.100 and 0.028 , respectively, after the artifact removal procedure. The CA is often characterized by having highly asymmetrical and super-Gaussian amplitude distributions, ${ }^{12}$ which are relatively easy to extract with cBSS or with HOS-BSS. As for the OAs, the SOS techniques may be better suited for this form of contamination, something that is in agreement with previous studies. ${ }^{33,34}$ By comparing Figs. 2 and 5, it is clear that some BSS-based artifact rejection procedures caused a reduction in the amount of low frequency power at the channels close to the eyes. Additionally, the number of peaks with extreme values also decreased notably. In the case of the PLN, the highest levels of removal were achieved with the ApEn and, specially, $P_{50}$ metrics almost independently on the BSS algorithm. When observing the results, it must be noted that the real reduction in power between 49 and $51 \mathrm{~Hz}$ is given by the total power measure. This is due to the fact that the normalized power depends on the amount of power in the whole frequency range, which is also affected by the removal of CA or OAs. Moreover, the actual reduction in peak at $50 \mathrm{~Hz}$ is higher than the ratios indicated in Tables 1 and 2 since these metrics account for the change in power in a $2 \mathrm{~Hz}$ band $(49$ to $51 \mathrm{~Hz}$ ). Actually, the power at $50 \mathrm{~Hz}$ is reduced about 30 times (i.e., about $14.7 \mathrm{~dB}$ ). All these findings suggest that none of the approaches is optimal for all forms of artifacts. Therefore, the selection of the strategy to reduce the contamination should rely on a priori knowledge about which is the most prominent artifact in the recordings to be cleaned.

A few publications have addressed the artifact removal (particularly the CA and OA) in MEGs with BSS. ${ }^{2,7,21,31,32}$ In most cases, the performance was only illustrated with a few visual examples. ${ }^{2,21,32}$ The quantification of the CA removal by Mantini et al. showed a ratio of the $A_{\mathrm{pp}}$ after and before the procedure of about 0.158 (computed averaging the results given in Ref. 31). Slightly better values than this one were found in this study (see Tables 1 and 2). Additionally, Dammers et al. reported rejection performances in terms of RMS of about 80 to $90 \%$ around the R-peaks and blinks located in reference channels. ${ }^{7}$ Nonetheless, the comparison of BSS artifact removal on the basis of previous studies is difficult due to the different databases. For this reason, we applied several approaches to a common and independent dataset of MEG signals.

Some limitations of this study deserve attention. The significance of results is limited by the size of the database (26 subjects). However, this size is similar to, or even larger than, that of other $\mathrm{EEG}^{30,35,36}$ and $\mathrm{MEG}^{2,7,12,31,32,40}$ studies. Additionally, the MEG activity was recorded from elderly people (mean age $=71.77$ years). In this age group, the identification of CA may be more challenging due to the possible presence of ventricular extrasystoles. However, the extension of our results to younger subjects might not be straightforward. In any case, our findings can directly be applied to elderly people. Additionally, by the own nature of MEG, the actual sources that originated the brain recordings are unknown. Hence, assessing the performance of the BSS is not straightforward as the separation cannot be absolutely validated. ${ }^{22,39}$ Yet, it is indeed possible to compare the brain signals before and after the artifact removal to gain quantitative information about how much artifactual activity has been rejected. ${ }^{7,12,31}$ However, further analyses, including a visual evaluation of the artifact removal, are needed to confirm that the most promising combinations of BSS algorithm, epoch length, and artifact detection metric did not remove brain activity. This is particularly the case for the OAs since some of the detection criteria might be sensitive to frontal brain activity. ${ }^{12}$ Moreover, the threshold of $\pm 2 \mathrm{pT}$ used in the evaluation of the artifact rejection may need to be changed if eventrelated activity is analyzed instead of background one. Nevertheless, recent results suggest that the BSS artifact removal does not denature the electromagnetic brain recordings. ${ }^{38}$ On the contrary, the ability of the EEG signals to distinguish control subjects from Alzheimer's Disease patients increased after the artifact removal. ${ }^{38}$

In sum, the objective assessment of the artifact removal in MEG background activity suggested the utility of several combinations of BSS algorithm and specific artifact detection metric. For its simplicity, the cBSS scheme is considered a very suitable approach to 
remove the $\mathrm{CA}$ as its performance ranked among the best in the reduction of this artifact. The $\mathrm{OA}$ reduction was the most difficult to evaluate. However, the evaluation results suggested that the combination of artifact detection metrics such as ApEn, $P_{\text {eyes }}$, and, specially, $P_{\text {If }}$ with SOS-BSS techniques was helpful to reduce the OA. Finally, the PLN was substantially removed by means of the specific metric $P_{50}$.

\section{ACKNOWLEDGMENTS}

This study was partially supported by the "Ministerio de Ciencia e Innovación" and FEDER grant TEC200802241. We are thankful to the three Reviewers of this manuscript for their useful comments.

\section{CONFLICT OF INTEREST}

There are no conflicts of interest that could inappropriately influence this manuscript.

\section{REFERENCES}

${ }^{1}$ Antervo, A., R. Hari, T. Katila, T. Ryhänen, and M. Seppänen. Magnetic fields produced by eye blinking. Electroencephalogr. Clin. Neurophysiol. 61(4):247-253, 1985.

${ }^{2}$ Barbati, G., C. Porcaro, F. Zappasodi, P. M. Rossini, and F. Tecchio. Optimization of an independent component analysis approach for artifact identification and removal in magnetoencephalographic signals. Clin. Neurophysiol. 115(5):1220-1232, 2004.

${ }^{3}$ Belouchrani, A., K. Abed-Meraim, J. F. Cardoso, and E. Moulines. A blind source separation technique using second-order statistics. IEEE Trans. Signal Process. 45(2): 434-444, 1997.

${ }^{4}$ Cardoso, J. F., and A. Souloumiac. Blind beamforming for non Gaussian signals. IEE Proc. F 140(6):362-370, 1993.

${ }_{5}^{5}$ Cichocki, A., S. Amari, K. Siwek, T. Tanaka, and A. Huy Phan. ICALAB for Signal Processing. November 2010. [online] http://www.bsp.brain.riken.jp/ICALAB/ICALAB SignalProc.

${ }^{6}$ Croft, R. J., and R. J. Barry. Removal of ocular artifact from the EEG: a review. Neurophysiol. Clin. 30(1):5-19, 2000.

${ }^{7}$ Dammers, J., M. Schiek, F. Boers, C. Silex, M. Zvyagintsev, U. Pietrzyk, and K. Mathiak. Integration of amplitude and phase statistics for complete artifact removal in independent components of neuromagnetic recordings. IEEE Trans. Biomed. Eng. 55(10):2353-2362, 2008.
${ }^{8}$ Delorme, A., and S. Makeig. EEGLAB: an open source toolbox for analysis of single-trial EEG dynamics including independent component analysis. J. Neurosci. Methods 134(1):9-21, 2004.

${ }^{9}$ Delorme, A., T. Sejnowski, and S. Makeig. Enhanced detection of artifacts in EEG data using higher-order statistics and independent component analysis. NeuroImage 34(4):1443-1449, 2007.

${ }^{10}$ Escudero, J., R. Hornero, and D. Abásolo. Consistency of the blind source separation computed with five common algorithms for magnetoencephalogram background activity. Med. Eng. Phys. 32(10):1137-1144, 2010.

${ }^{11}$ Escudero, J., R. Hornero, D. Abásolo, and A. Fernández. Blind source separation to enhance spectral and non-linear features of magnetoencephalogram recordings. Application to Alzheimer's disease. Med. Eng. Phys. 31(7):872-879, 2009.

${ }^{12}$ Escudero, J., R. Hornero, D. Abásolo, A. Fernández, and M. López-Coronado. Artifact removal in magnetoencephalogram background activity with independent component analysis. IEEE Trans. Biomed. Eng. 54(11):1965-1973, 2007.

${ }^{13}$ Escudero, J., R. Hornero, J. Poza, D. Abásolo, and A. Fernández. Assessment of classification improvement in patients with Alzheimer's disease based on magnetoencephalogram blind source separation. Artif. Intell. Med. 43(1):75-85, 2008.

${ }^{14}$ Fatourechi, M., A. Bashashati, R. K. Ward, and G. E. Birch. EMG and EOG artifacts in brain computer interface systems: a survey. Clin. Neurophysiol. 118(3):480-494, 2007.

${ }^{15}$ Fitzgibbon, S. P., D. M. W. Powers, K. J. Pope, and C. R. Clark. Removal of EEG noise and artifact using blind source separation. J. Clin. Neurophysiol. 24(3):232-243, 2007.

${ }^{16}$ Gävert, H., J. Hurri, J. Särelä, and A. Hyvärinen. FastICA Toolbox. November 2010. [online] http://www.cis.hut.fi/ projects/ica/fastica.

${ }^{17}$ Greco, A., N. Mammone, F. C. Morabito, and M. Versaci. Kurtosis, Renyi's entropy and independent component scalp maps for the automatic artifact rejection from eeg data. Int. J. Signal Process. 2(4):240-244, 2006.

${ }^{18}$ Hämäläinen, M., R. Hari, R. J. Ilmoniemi, J. Knuutila, and O. V. Lounasmaa. Magnetoencephalography-theory, instrumentation, and applications to noninvasive studies of the working human brain. Rev. Mod. Phys. 65(2):413-497, 1993.

${ }^{19}$ Huang, D. S., and J. X. Mi. A new constrained independent component analysis method. IEEE Trans. Neural Netw. 18(5):1532-1535, 2007.

${ }^{20}$ Hyvärinen, A., J. Karhunen, and E. Oja. Independent Component Analysis. Hoboken, NJ: John Wiley \& Sons, 2001.

${ }^{21}$ James, C. J., and O. J. Gibson OJ. Temporally constrained ICA: an application to artifact rejection in electromagnetic brain signal analysis. IEEE Trans. Biomed. Eng. 50(9): 1108-1116, 2003.

${ }^{22}$ James, C. J., and C. W. Hesse. Independent component analysis for biomedical signals. Physiol. Measure. 26(1): R15-R39, 2005.

${ }^{23}$ Jousmäki, V., and R. Hari. Cardiac artifacts in magnetoencephalogram. J. Clin. Neurophysiol. 13(2):172-176, 1996.

${ }^{24}$ Joyce, C. A., I. F. Gorodnitsky, and M. Kutas. Automatic removal of eye movement and blink artifacts from EEG 
data using blind component separation. Psychophysiology 41(2):313-325, 2004.

${ }^{25}$ Jung, T. P., S. Makeig, C. Humphries, T. W. Lee, M. J. Mckeown, V. Iragui, and T. J. Sejnowski. Removing electroencephalographic artifacts by blind source separation. Psychophysiology 37(2):163-178, 2000.

${ }^{26}$ Lee, T. W., M. Girolami, and T. J. Sejnowski. Independent component analysis using an extended infomax algorithm for mixed subgaussian and supergaussian sources. Neural Comput. 11(2):417-441, 1999.

${ }^{27}$ LeVan, P., E. Urrestarazu, and J. Gotman. A system for automatic artifact removal in ictal scalp EEG based on independent component analysis and Bayesian classification. Clin. Neurophysiol. 117(4):912-927, 2006.

${ }^{28} \mathrm{Li}$, Y., Z. Ma, W. Lu, and Y. Li. Automatic removal of the eye blink artifact from EEG using an ICA-based template matching approach. Physiol. Measure. 27(4):425-436, 2006.

${ }^{29} \mathrm{Lu}$, W., and J. C. Rajapakse JC. Approach and applications of constrained ICA. IEEE Trans. Neural Netw. $16(1): 203-212,2005$.

${ }^{30}$ Mammone, N., and F. C. Morabito. Enhanced automatic artifact detection based on independent component analysis and Renyi's entropy. Neural Netw. 21(7):1029-1040, 2008.

${ }^{31}$ Mantini, D., R. Franciotti, G. L. Romani, and V. Pizzella. Improving MEG source localizations: An automated method for complete artifact removal based on independent component analysis. NeuroImage 40(1):160-173, 2008.

${ }^{32}$ Okada, Y., J. Jung, and T. Kobayashi. An automatic identification and removal method for eye-blink artifacts in event-related magnetoencephalographic measurements. Physiol. Measure. 28(12):1523-1532, 2007.
${ }^{33}$ Romero, S., M. A. Mañanas, and M. J. Barbanoj. A comparative study of automatic techniques for ocular artifact reduction in spontaneous EEG signals based on clinical target variables: a simulation case. Comput. Biol. Med. 38(3):348-360, 2008.

${ }^{34}$ Romero, S., M. A. Mañanas, and M. J. Barbanoj. Ocular reduction in EEG signals based on adaptive filtering, regression and blind source separation. Ann. Biomed. Eng. 37(1):176-191, 2009.

${ }^{35}$ Shao, S., K. Shen, C. Ong, E. Wilder-Smith, and X. Li. Automatic EEG artifact removal: a weighted support-vector-machine approach with error correlation. IEEE Trans. Biomed. Eng. 56(2):336-344, 2008.

${ }^{36}$ Ting, K. H., P. C. W. Fung, C. Q. Chang, and F. H. Y. Chan. Automatic correction of artifact from single-trial event-related potentials by blind source separation using second order statistics only. Med. Eng. Phys. 28(8):780 794, 2006

${ }^{37}$ Tong, L., R. W. Liu, V. C. Soon, and Y. F. Huang. Indeterminacy and identifiability of blind identification. IEEE Trans. Circuits Syst. 38(5):499-509, 1991.

${ }^{38}$ Vialatte, F. B., J. Solé-Casals, M. Maurice, C. Latchoumane, N. Hudson, S. Wimalaratna, J. Jeong, and A. Cichocki. Improving the quality of EEG data in patients with Alzheimer's disease using ICA. Lect. Notes Comput. Sci. 5507:979-986, 2009.

${ }^{39}$ Vigário, R., and E. Oja. BSS and ICA in neuroinformatics: from current practices to open challenges. IEEE Rev. Biomed. Eng. 1:50-61, 2008.

${ }^{40}$ Zavala Fernández, H., T. H. Sander, M. Burghoff, R. Orglmeister, and L. Trahms. Comparison of ICA algorithms for the isolation of biological artifacts in magnetoencephalography. Lect. Notes Comput. Sci. 3889:511518,2006 